\title{
Effectiveness of Mesotherapy on Temporomandibular
} Joint Disorders

\author{
André Pedro Oliveira Cruz ${ }^{*}$, Ana Filipa Rodrigues das Neves, Isabel Ramires and Manuel Mendonça \\ Serviço de Medicina Física e de Reabilitação, Hospital de S. José, Centro Hospitalar de Lisboa Central, Lisboa 1150-199, Portugal
}

\begin{abstract}
TMJDs (Temporomandibular joint disorders) are a common pathology but best treatment remains unclear. The goal of this study was to evaluate the effectiveness of mesotherapy on TMJD. We conducted a case review of all TMJD patients treated since 2006 with mesotherapy procedures only and using a cocktail of drugs composing of lidocaine, piroxicam and pentoxifylline. Patients were treated every 15 days until symptomatic relief was achieved and thereafter every two months. Main outcome was complete symptomatic relief, and associated factors were analysed. 27 patients were included. Complete pain relief was achieved in 26 . The necessary number of procedures to get pain relief ranged from 1 to 6 (median of 1 ) and the total number of treatments from 1 to 40 (median of 11). 19 patients had other painful complains mainly related with anxiety disorders. A weak correlation $(r=0.357 ; p=$ 0.05 ) between anxiety disorders and the required months of treatments was found. Mesotherapy is a valuable option on pain relief in a temporomandibular pain syndrome with none of the systemic effects of oral medication. Anxiety may have a role on the aetiology and therapeutic success highlights the holistic approach of these patients. Future comparative studies are necessary.
\end{abstract}

Key words: TMJDs (Temporomandibular joint disorders), mesotherapy, pain.

\section{Introduction}

TMJDs (Temporomandibular joint disorders) are a fairly common pathology. It is described that up to $40 \%$ of general population can present at least one sign of TMJDs [1]. In adults, this percentage can be even higher and $75 \%$ that may have one sign of joint dysfunction, but the majority doesn't report any symptom and only $10 \%-20 \%$ will seek for treatment [1-3].

The etiology of TMJD remains unclear but it seems to be caused by multiple factors, related to the muscles or the joint itself. Para functional habits (bruxism, lip biting or teeth grinding) $[4,5]$, anxiety, intra-articular disc or capsule damage and abnormal dental occlusion may be involved on the responsible mechanism of TMJD. However a lot of people with these characteristics will never develop symptoms [1].

Articular disarrangements usually produce joint

*Corresponding author: André Pedro Oliveira Cruz, PM\&R trainee at Serviço de Medicina Física e de Reabilitação, Hospital de São José - Centro Hospitalar Lisboa Central, Lisboa, Portugal, research field: minnimally invasive techniques. E-mail: andrepocruz@gmail.com. clicking or grating while headache, neck pain or painful movements of jaw are more associated to muscular malfunctions. Most common symptoms are jaw pain, limited jaw movement, clicking or grating joint, neck pain and headache [6]. It is described that up to $50 \%$ of patients with chronic headache referred to a neurologist have signs or symptoms of TMJD [7]. Consequently pain related complains (neck, head, TMJ) will be one of the most important and prevalent problem in persons with TMJD. In this aspect woman seems to have higher and more severe pain prevalence [6, 8-10]. Psychological abnormalities have been also associated with elevated pain grade $[8$, 9, 11]. In this context, though most of TMJ patients will remain asymptomatic or have symptoms during a short time $[1,12]$ pain relief will be one of the main goals of TMJD treatment. MRI (Magnetic resonance imaging) is considered to be the gold standard for diagnose TMJ problems [13]. Conservative therapies remain as the first line management [14-19]. In some cases, when problems perpetuate, surgery repair may be required [20]. 
Mesotherapy is a minimally invasive technique that consists on intradermal or subcutaneous injections of one or various drugs [21]. It has been described that in mesotherapy the skin can act as a natural time-release system [22].The procedure will only require a syringe and a needle (commonly $30 \mathrm{G}$ ) or it could be performed using a mesotherapy gun (Fig. 1). The necessary number of injections is variable and proportional to the area to treat, but the necessary dose is relatively lower than the needed by other methods. A lot of pathologies seem to benefit from this technique like tendinopathies, enthesopathies, fasciitis, muscular stretches, miofascial disorders, osteoarthritis and many others [23-30]. According to our knowledge there are no studies reporting the treatment effects of this technique in TMJD. The goal of this study was to evaluate the effectiveness of mesotherapy as a viable option for TMJD.

\section{Contents}

We conducted a case review of all TMJD patients treated in a central hospital from 2006 until 2013 with mesotherapy procedures. During the course of treatment it was ensured that all patients only performed this treatment modality. Mesotherapy was used to introduce pharmaceutical drugs via multiple intradermal injections.

Mesotherapy consultation in Hospital de S. José started 25 years ago and every year around 2,500 patients, that present pain from the muscle skeleton system, benefit from this treatment modality. In the last decade we had tried to respond to some patients scheduled in our consultation by other pathologies that asked for help on their temporomandibular complaints. Sample was formed by these patients and other that were referred from other specialties that had not been well succeeded in treatment.

Due to the theoretical inflammatory mechanism of the pain, the use of common drug compositions was started in TMJD patients as applied in other similar pathologies. We have tried different types of analgesic, anti-inflammatory and vasodilator drugs and with different proportions, but in the last years before our study we noticed that one specific formulation has achieved the best results. This liquid mixture was formed by a non-steroid anti-inflammatory (piroxicam $20 \mathrm{mg} / \mathrm{mL}$ ), a local anesthetic (lidocaine at $1 \%$ ) and a vasodilator (pentoxifylline $20 \mathrm{mg} / \mathrm{mL}$ ).

The procedures were performed by a Physical Medicine and Rehabilitation specialist or trainee and under appropriate sterile precautions. Most patients were treated in seated position and only few requested decubitus. In order to provide a superficial analgesia we used ethyl chloride. To achieve a more precise and volume controlled injection we use a mesotherapy gun. In this way, we performed 6-8 injections of $0.1 \mathrm{cc}$ each, using a total of $0.6-0.8 \mathrm{cc}$. We established the time between appointments as for other similar pathologies: patients were treated every 15 days until symptomatic relief and thereafter every 2 months. We measured the necessary number of treatments to attain some pain relief and the absence of pain (VAS (Visual analogue scale) grade 0). Patients were followed and we evaluated if the response was uninterrupted. Main outcome of this study was the complete symptomatic relief. Some associated factors like presence of psychiatric disorders or other painful complains, gender, age, traumatic history and affected side were analysed. Description of procedure and evaluation of

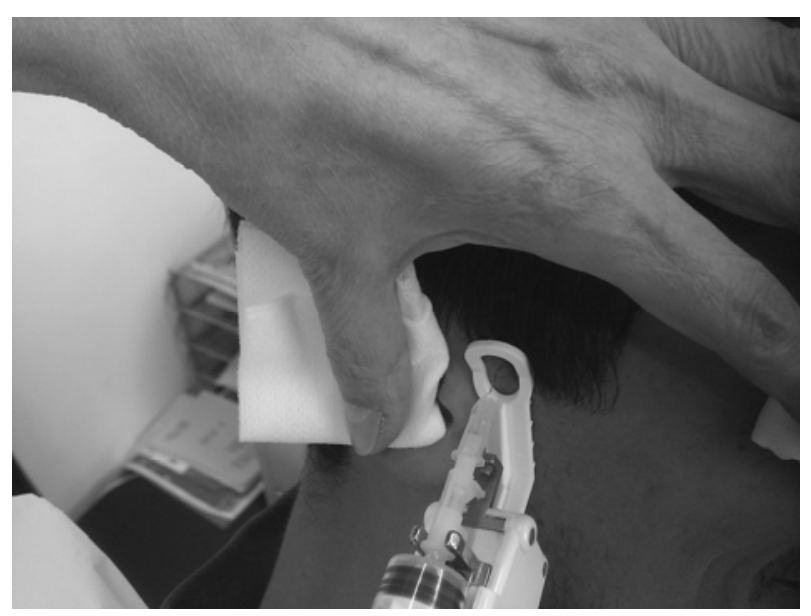

Fig. 1 Mesotherapy application on temporomandibular area. 
possible side-effects during and after it had been registered. Patients had been informed and agreed to participate in the study.

\subsection{Statistical Analysis}

Qui-square and fisher exact tests were used for analysis (SPSS 19.0, Chicago, IL, USA), considering $95 \%$ significance level $(\mathrm{p}<0.05)$. For a correlation analysis, Spearman correlation coefficients were calculated. Confidence interval for the rate of success of treatment was calculated using OpenEpi ${ }^{\circledR}$. As this was an exploratory pilot experimental study, $\mathrm{p}$-values between 0.05 and 0.10 were reported.

\section{Results}

A total of 27 patients were included in this study. The majority 14 started treatment at 2007 and ever since 2 new persons were included each year. Twenty four were female and only three were men. The age ranged from 25 to 80 years old (mean of 52.92) and no age predominance was found. Six patients had TMJ trauma history like fracture, dislocation or surgery (Fig. 2). Bilateral affection was present in 8 patients and 10 had only left side affected. Total treatment period ranged from only one application to several applications during an 84 months period after initial treatment (median of 19). Complete pain relief (VAS - 0) was achieved in 26 of 27 patients $(96 \%$; CI95\% 83.1-99.8) (Fig. 3). The necessary number of procedures to get some pain relief ranged from 1 to 6 (median of 1). 19 patients had achieved benefit at the first application and only 3 needed more than 3 treatments to notice some difference (Fig. 4). It is rewarded to find that 6 patients had full benefit with just one application and that 18 patients had full benefit with less than ten sessions. The total number of treatments ranged from 1 to 40 (median of 11). 19 patients had other painful complains, mainly related with anxiety disorders. The most associated pathologies were anxiety, fibromyalgia and depression (Fig. 5). A weak correlation (Spearman's rho $\mathrm{r}=$
$0.357 ; \mathrm{p}=0.05$ ) between anxiety disorders and the required months of treatments was found.

\section{TMJ trauma history}

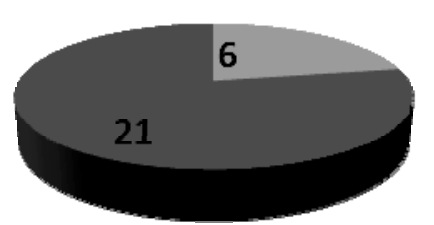
Fracture, dislocation, surgery...
Non trauma history

Fig. 2 Trauma history on study TMJ patients.

\section{Treatment response}

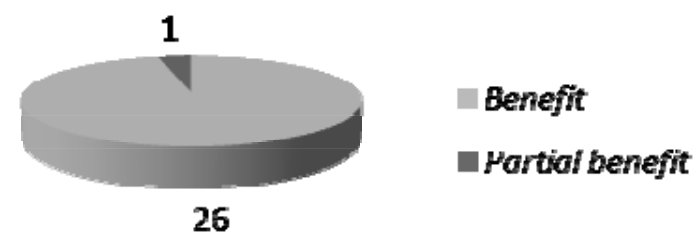

Benefit rate $96 \%$ (95\% CI 83.1-99.8)

Fig. 3 Treatment response for TMJD under mesotherapy technique.

Number of treatments to achieve symptomatic relief

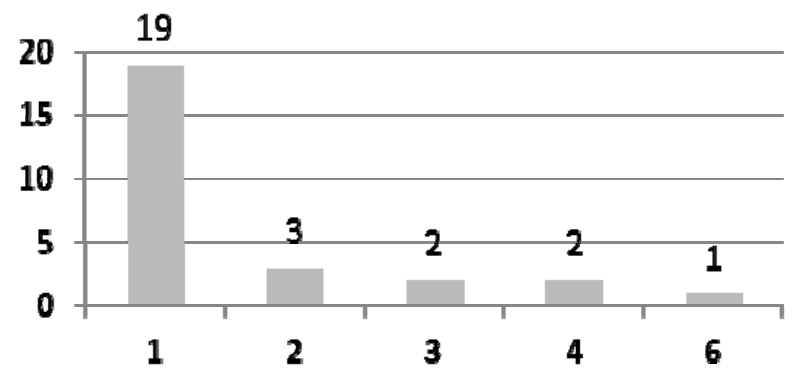

Fig. 4 Number of mesotherapy treatments for TMJD to achieve symptomatic relief.

\section{Anxiety disorders antecedents}

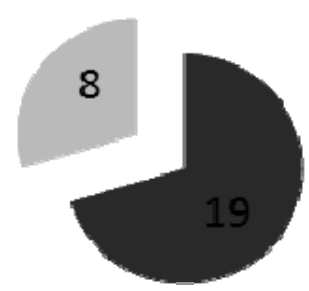

FM;

Depresion;

other

Non A.D

Fig. 5 Anxiety disorders on study TMJ patients. 
We have not found an association between affected side, traumatic history, age, sex and response to treatment.

The total cost of the procedure including sterile materials, mesotherapy kit and drugs was less than $10 €$. Time to perform each treatment was approximately 20 minutes.

The procedure was well tolerated by all patients, with only slight pain complaints during the injection, despite the previous application of ethyl chloride to avoid pain. During and after the treatments we did not register any side-effect or complications. After achieving the absence of pain complains, the results had been persistent.

\section{Discussion}

TMJD are very commons on general population but the majority of affected persons are asymptomatic, self-limited or reveal slight symptoms [31-33]. Chronic and resistant to treatment temporomandibular disorders will consume a lot of appointments and clinical resources. Mesotherapy treatment modality seems to offer a huge benefit to these patients, acting on pain complaints and on the healing process. While our mesotherapy appointments are a representative amount in hospital care of around 2,000 patients per year, the number of patients in this study was very small, mainly due to small fraction of patients with TMJD that will seek for medical help and because we are not a specialized appointment for temporomandibular care. Although the small sample group, results are very interesting if we consider that only one patient has not found full benefit and had persistence of pain.

In our mesotherapy appointments the majority are females and elderly patients as expected, and this aspect may have influence in our sample.

Concerning to the mixture, other formulation have been tried in the past but results were not so good and persistent. That's the main reason why we performed this study under this tree drugs. From our knowledge few publications related to mesotherapy techniques are available and we haven't found any work analyzing the effects of mesotherapy on TMJD or a recommended mixture. The composition described above seems to be the best option till now but it is possible that, in the future, other associations can achieve similar or better results.

Treatment costs are also an important consideration for practitioners and their patients. Although a cost benefit analysis has not been done, assuming usual medication and material pricing (aprox. $10 €$ ), when compared to other available treatments (i.e.: oral anti-inflammatory medication, muscle relaxants, physiotherapy, splints, laser, ultrasound, chirurgical procedures, others) mesotherapy seems to be a reasonable alternative to treat TMJD.

Finally, our study was not randomized and lack of a study group control. It will be interesting to try this management in a larger sample.

\section{Conclusions}

Pathology from temporomandibular joint is very common and a lot of data in scientific literature can be found. While majority of affected patients will not show or have auto-limited complaints, the best treatment option for chronic and severe patients is not completely established. A lot of treatments had been applied in the past from non-invasive (mobilization, splint, physical modalities and many others) to surgical repair with variable success and does not exist a standard treatment [16-19].

Mesotherapy technique is an intradermal route to apply different drugs. Till now, few studies had been published about mesotherapy method and no study about its effects on TMJD [23-30]. A lot of aspects should encourage the use of this method. An important aspect was the security and success of results. The cheap costs of the procedure can be a very important detail in our times. Other important aspects are the easy way and the short time to perform it. Comparing to other treatment modalities, mesotherapy can be cheaper, faster, more secure and well succeed. For all 
these motives, mesotherapy may be a good treatment option to these patients. Further studies are essentials to evaluate these effects.

\subsection{Competing Interests}

The authors declared no competing interest with respect to the authorship and/or publication of this article.

\subsection{Authors' Contributions}

AC participated in conception and design, acquisition of data, analysis and interpretation of data; AN participated in acquisition and interpretation of data; IR and MM participated in conception and design, analysis and interpretation of data. All authors read and approved the final manuscript.

\subsection{Acknowledgments}

The authors wish to thank the Epidemiology and Statistics Office of the Research Unit, Centro Hospitalar de Lisboa Central, for the help provided in data analysis and for review and criticism in improving the manuscript.

The paper was presented at the $10^{\text {th }}$ Mediterranean Congress of Physical and Rehabilitation Medicine (2013).

\subsection{Funding}

No financial support for this research was received.

\section{References}

[1] Buescher, J. J. 2007. "Temporomandibular Joint Disorders.” Am Fam Physician 76 (10): 1477-82.

[2] Koh, H. and Robinson, P. G. 2004. "Occlusal Adjustment for Treating and Preventing Temporomandibular Joint Disorders." J Oral Rehabil. 31: 287-92.

[3] Rutkiewicz, T., Kononen, M., Suominen-Taipale, L., Nord-blad, A. and Alanen, P. 2006. "Occurrence of Clinical Signs of Temporomandibular Disorders in Adult Finns." J Orofac Pain.20: 208-17.

[4] Okeson, J. P. 1996. "For the American Academy of Orofacial Pain. Orofacial Pain: Guidelines for Assessment, Diagnosis and Management." Chicago, Ill. Quintessence Pub.
[5] Santana-Mora, U., López-Cedrún, J., Mora, M., Otero, X. and Santana-Penín, U. 2013. "Temporomandibular Disorders: The Habitual Chewing Side Syndrome." PLoS One 8 (4): 59980.

[6] Scrivani, S., Keith, D. and Kaban, L. 2008. “Temporomandibular Disorders." N Engl J Med 359: 693-705.

[7] Wright, E. F., Clark, E. G., Paunovich, E. D. and Hart, R. G. 2006. "Headache Improvement through TMD Stabilization Appliance and Self-Management Therapies." Cranio. 24: 104-11.

[8] Blanco-Hungría, A., Rodríguez-Torronteras, A., Blanco-Aguilera, A., Biedma-Velázquez, L., Serrano-del-Rosal, R. and Segura-Saint-Gerons, R. et al. 2012. "Influence of Sociodemographic Factors upon pain Intensity in Patients with Temporomandibular Joint Disorders Seen in the Primary Care Setting." Med Oral Patol Oral Cir Bucal. 17 (6): 1034-41.

[9] Dougall, A., Jimenez, C., Haggard, R., Stowell, A., Riggs, R. and Gatchel, R. 2012. "Biopsycholosocial Factors Associated with Subcategories of Acute Temporomandibular Joint Disorders." J Orofac Pain. 26 (1): 7-16.

[10] Bagis, B., Ayaz, E., Turgut, S., Durkan, R. and Özcan, M. 2012. "Gender Difference in Prevalence of Signs and Symptoms of Temporomandibular Joint Disorders: A Retrospective Study on 243 Consecutive Patients." Int $J$ Med Sci. 9 (7): 539-44.

[11] Rugh, J. D. and Solberg, W. K. 1976. "Psychological Implications in Temporomandibular Pain and Dysfunction." Oral Sci Rev.7: 3-30.

[12] Weiss, P. F., Arabshahi, B., Johnson, A., Bilaniuk, L. T., Zarnow, D. and Cahill, A. M. et. al. 2008. "High Prevalence of Temporomandibular Joint Arthritis at Disease Onset in Children with Juvenile Idiopathic Arthritis, as Detected by Magnetic Resonance Imaging but not by Ultrasound." Arthritis Rheum. 58 (4): 1189-96.

[13] Kulekcioglu, S., Sivrioglu, K., Ozcan, O. and Parlak, M. 2003. "Effectiveness of Low-Level Laser Therapy in Temporomandibular Disorder." Scand. J. Rheumatol. 32: 114-8.

[14] Brown, C. R. 1998. "Temporomandibular Joint Dysfunction.” In Windsor RE, Lox DM, eds. Soft Tissue Injuries: Diagnosis and Treatment. Philadelphia, Hanley \& Belfus. 225-41.

[15] McNeill, C. 1997. "Management of Temporomandibular Disorders: Concepts and Controversies." J Prosthet Dent 77: 510-22.

[16] Samiee, A., Sabzerou, D., Edalatpajouh, F., Clark, G. and Ram, S. 2011. "Temporomandibular Joint Injection with Corticosteroid and Local Anesthetic for Limited Mouth Opening." Journal of Oral Science 53 (3): 321-25. 
[17] Özkan, F., Çakir, Ö. and N, Erkorkmaz, U. 2011. "Trigger Point Injection Therapy in the Management of Myofascial Temporomandibular Pain." $A \breve{G R I ~} 23$ (3): $119-25$.

[18] Vicente-Barrero, M., Yu-Lu, S., Zhang, B., Bocanegra-Pérez, S., Durán-Moreno, D. and López-Márquez, A. et al. 2012. "The Efficacy of Acupuncture and Decompression Splints in the Treatment of Temporomandibular Joint Pain-Dysfunction Syndrome." Med Oral Patol Oral Cir Bucal. 17 (6): 1028-33.

[19] Dostalová, T., Hlinakova, P., Kasparova, M., Rehacek, A., Vavrickova, L. and Navrátil, L. 2012. "Effectiveness of Physiotherapy and GaAlAs Laser in the Management of Temporomandibular Joint Disorders." Photomed Laser Surg. 30 (5): 275-80.

[20] American Society of Temporomandibular Joint Surgeons. 2003. "White Paper: Guidelines for Diagnosis and Management of Disorders Involving the Temporomandibular Joint and Related Musculoskeletal Structures." Cranio 21: 68-76.

[21] Sivagnanam, G. 2010. "Mesotherapy-The French Connection." J Pharmacol Pharmacother. 1 (1): 4-8.

[22] Kaplan, A. R. 1985. "Devenir d'un Produit Marque Injecte par Quatre Voies Differentes.” Bulletin SFM. 62.

[23] Mammucari, M., Gatti, A., Maggiori, S. and Sabato, A. 2012. "Role of Mesotherapy in Musculoskeletal Pain: Opinions from the Italian Society of Mesotherapy." Evid Based Complement Alternat Med. 1-12.

[24] Mammucari, M., Gatti, A., Maggiori, E., Vergari, B., Gafforio, P. and Tufaro, G. et al. 2013. "Informed Consent and Experimental Treatments: the Case of Mesotherapy." Recenti Prog Med. 104 (5): 214-7.

[25] Baspeyras, M., Rouvrais, C., Liégard, L., Delalleau, A., Letellier, S. and Bacle, I. et al. 2013. "Clinical and
Biometrological Efficacy of a Hyaluronic Acid-Based Mesotherapy Product: a Randomised Controlled Study."Arch Dermatol Res. 305 (8): 673-82.

[26] El-Domyati, M., El-Ammawi, T. S., Moawad, O., El-Fakahany, H., Medhat, W. and Mahoney, M. G. et al. 2012. "Efficacy of Mesotherapy in Facial Rejuvenation: a Histological and Immunohistochemical Evaluation." Int $J$ Dermatol 51 (8): 913-9.

[27] Al-Qarqaz, F., Al-Aboosi, M., Al-shiyab, D. and Al Dabbagh, Z. 2012. "Using Cold Air for Reducing Needle-Injection Pain.” Int J Dermatol. 51 (7): 848-52.

[28] Herreros, F. O., Moraes, A. M. and Velho, P. E. 2011. "Mesotherapy: a Bibliographical Review." An Bras Dermatol 86 (1): 96-101.

[29] Sarkar, R., Garg, V. K. and Mysore, V. 2011. "Position Paper on Mesotherapy." Indian J Dermatol Venereol Leprol 77 (2): 232-7.

[30] Di Cesare, A., Giombini, A., Di Cesare, M., Ripani, M., Vulpiani, M. C. and Saraceni, V. M. 2011. "Comparison between the Effects of Trigger Point Mesotherapy versus Acupuncture Points Mesotherapy in the Treatment of Chronic Low Back Pain: a Short Term Randomized Controlled Trial." Complement Ther Med. 19 (1): 19-26.

[31] Tallents. R. H., Hatala, M., Katzberg, R. W. and Westesson, P. L. 1993. "Temporomandibular Joint Sounds in Asymptomatic Volunteers." The Journal of prosthetic dentistry 69 (3): 298-304.

[32] Garcia, A., Juim, P., Goiato, M., Santos, P., Ribeiro, A. and Pita, M. et al. 2008. "Effect of Occlusion on Joint Sounds in Asymptomatic Individuals." Acta Odontológica Latinoamericana: AOL 21 (2): 135-40.

[33] Isberg, A. 2001. "Growth Changes." In: Isberg, A. (Ed.) Temporomandibular Joint Dysfunction: A Practitioner's Guide. Taylor \& Francis, London, UK. 145-58. 